\title{
Local Two-Body Parent Hamiltonians for the Entire Jain Sequence
}

\author{
Sumanta Bandyopadhyay ${ }^{1,2}$ Gerardo Ortiz ${ }^{3,}, 1$ Zohar Nussinov, ${ }^{1}$ and Alexander Seidel ${ }^{1}$ \\ ${ }^{1}$ Department of Physics, Washington University, St. Louis, Missouri 63130, USA \\ ${ }^{2}$ Nordita, KTH Royal Institute of Technology and Stockholm University, Roslagstullsbacken 23, SE-106 91 Stockholm, Sweden \\ ${ }^{3}$ Department of Physics, Indiana University, Bloomington, Indiana 47405-7105, USA
}

(Received 6 November 2019; accepted 27 April 2020; published 15 May 2020)

\begin{abstract}
Using an algebra of second-quantized operators, we develop local two-body parent Hamiltonians for all unprojected Jain states at filling factor $n /(2 n \mathrm{p}+1)$, with integer $n$ and (half-)integer $\mathrm{p}$. We rigorously establish that these states are uniquely stabilized and that zero mode counting reproduces mode counting in the associated edge conformal field theory. We further establish the organizing "entangled Pauli principle" behind the resulting zero mode paradigm and unveil an emergent $\mathrm{SU}(n)$ symmetry characteristic of the fixed point physics of the Jain quantum Hall fluid.
\end{abstract}

DOI: $10.1103 /$ PhysRevLett.124.196803

Introduction.-The fractional quantum Hall $(\mathrm{FQH})$ effect enjoys a unique position in strongly correlated electron physics both as a fascinating physical effect [1] as well as a central juncture for the percolation of ideas between correlated electron physics and other areas of theoretical and mathematical physics. Originally, the success of the field owes much to construction principles for variational wave functions [2-6] and associated ideas to connect the latter to effective field theories [5,7-9]. In our opinion, the intimacy of the connection between microscopics and effective quantum field theory that is achievable in this field is, in some cases, essentially unparalleled. This is the case when the construction of a parent Hamiltonian $[4,5,10,11]$ is possible that falls into what we term the "zero mode (ZM) paradigm": The zero (energy) mode space of a positive semidefinite Hamiltonian is composed of an incompressible state as well as edge or quasihole excitations, where the counting of ZMs in each angular momentum sector (relative to the incompressible state) precisely matches $[6,12,13]$ the mode counting in the conformal edge theory. This then unambiguously points to the edge conformal field theory associated to the state, and, thanks to bulk-edge correspondence, all universal physics are then essentially fixed through exact properties of the microscopic Hamiltonian.

While a considerable number of these very special Hamiltonians exist, they are absent for many phases that are of central importance to the theory of the Hall effect. The latter include almost all phases described by Jain composite fermion (CF) states [14-17], which are key to

Published by the American Physical Society under the terms of the Creative Commons Attribution 4.0 International license. Further distribution of this work must maintain attribution to the author(s) and the published article's title, journal citation, and DOI. Funded by Bibsam. the understanding of the physics at Landau level (LL) filling factor $\nu<1$. While some Hamiltonians have been proposed for (non-Laughlin) Jain-type states [18-20], a ZM paradigm has only been established at 2/5 in Ref. [21] (for other more exotic parton states in Ref. [22]). There, some of us have argued that such a paradigm is possible in principle only for unprojected Jain states, which are well known to be in the same phase as their projected counterparts $[18,23]$. In this case, traditional first-quantized construction principles for parent Hamiltonians face unusual challenges. The latter seek to enforce "analytic clustering properties" [24-26] in the few-body density matrices of ZMs $[4,5,10,11,27,28]$. Indeed, unprojected Jain states generally have a zero of order $2 p+1$, with (half-)integer $p \geq 0$, when two particles meet at the same point. However, enforcing just this $(2 p+1)$-clustering property will generally lead to more exotic "parton" states $[3,7,29]$ as the incompressible ground states when more than $n=2$ LLs are present $[22,30]$. Actually, the $(2 p+1)$-clustering property comes from a purely holomorphic factor of the wave function while an antiholomorphic dependence is also present. This additional information is not straightforwardly enforced through a local Hermitian few-body interaction.

In this work, we solve this problem for all Jain CF states at filling factors $n /(2 n p+1)$, with integer $n \geq 1$. We utilize a recently developed operator formalism [31] that describes CFs as second-quantized objects in Fock space. This leads to an algebraic construction of the parent Hamiltonian that represents a radical departure from the traditional constructions principles described above, and fully embraces the "guiding-center-only" approach to FQH physics that has recently been influential [32,33]. Our results have further important ramifications for the theory of frustration free lattice Hamiltonians, in that we establish a framework where these become tractable even if interactions are not strictly short-ranged in generalized lattice 
coordinates. A close connection with the recently celebrated matrix-product structure of many $\mathrm{FQH}$ states [34-36] is anticipated, though we leave details for future work [37].

Composite fermions and zero modes. - The unprojected (mixed-LL) Jain state at filling factor $\nu=n /(2 n \mathrm{p}+1)$ [38] can be defined in disk geometry as

$$
\Psi_{n, \mathrm{p}}(N)=\prod_{1 \leq i<j \leq N}\left(z_{i}-z_{j}\right)^{2 \mathrm{p}} \Phi_{n}(N),
$$

where $\Phi_{n}(N)$ denotes an integer quantum Hall (IQH) state of $N$ particles in $n$ LLs, and the $z_{i}=x_{i}+i y_{i}, \bar{z}_{i}=x_{i}-i y_{i}$ are the particles' complex coordinates. $\Phi_{n}(N)$ is by definition a state of "densest" possible electron configuration for given $n$ and $N$, where ambiguities at the edge may arise for certain $N$ that we will resolve below.

Equation (1) clearly has a "clustering property," where the wave function has a $(2 p+1)$ th order zero when two particles converge to the same point. However, only for $n=2$ [21,22] does Eq. (1) represent the densest (largest filling factor) wave function(s) having this property. Related to that, for $n=2, \mathrm{p}=1$ there is the aforementioned, well-documented parent Hamiltonian satisfying the zero mode paradigm. To solve the general problem, we turn to an alternative characterization given by some of us [31] in terms of an algebra of second-quantized operators, which can be understood as "ZM generators." We begin by summarizing the nuts and bolts of this formalism.

In first quantization, an orbital $\phi_{m, \ell}$ in the $(m+1)$ th LL, $m=0,1, \ldots$, with angular momentum $\ell$ is a superposition of monomials of the form $\mu_{a, \ell}=\bar{z}^{a} z^{\ell+a}$, with $0 \leq a \leq m$. (We omit obligatory Gaussian factors.) Higher LL manybody wave functions such as Eq. (1) may be expanded in $\mu_{a, \ell}$, adorned with additional particle indices. A significant advantage of the first-quantized presentation is the fact that this expansion is essentially geometry independent, assuming that we limit ourselves to zero genus geometries (disk, cylinder, sphere) [33]. This is so since there is a one-to-one correspondence between the wave functions in these geometries, once $\bar{z}, z$ (for the disk) are replaced with suitable functions of coordinates respecting the boundary conditions of the respective geometries. In other words, variational wave functions such as Eq. (1) are described by the same polynomials in the genus 0 geometries. To obtain a manifestly geometry independent language, and to the extent that the successful construction of a parent Hamiltonian is a direct consequence of the underlying polynomial structure, however complicated, it proves advantageous to make the monomials $\mu_{a, \ell}$ the essential degrees of freedom of the second-quantized formalism also. For fixed $a$, we think of these orbitals as constituting a " $\Lambda$ level" $(\Lambda \mathrm{L})$. We thus introduce pseudofermion [39] operators $\tilde{c}_{a, \ell}, \tilde{c}_{a, \ell}^{*}$ satisfying canonical anticommutation relations,

$$
\left\{\tilde{c}_{a, \ell}, \tilde{c}_{a^{\prime}, \ell^{\prime}}^{*}\right\}=\delta_{a, a^{\prime}} \delta_{\ell, \ell^{\prime}},
$$

where $\tilde{c}_{a, \ell}^{*}$ creates an electron in the orbital $\mu_{a, \ell}$. These orbitals are not normalized or orthogonal (for fixed $\ell$ ), and hence, $\tilde{c}_{a, \ell}^{*}$ and $\tilde{c}_{a, \ell}$ are not Hermitian conjugates, but this will present no obstacle in the following. If desired, at the end we may always return to the canonical creation and annihilation operators $c_{m, \ell}, c_{m, \ell}^{\dagger}$ of the orbitals $\phi_{m, \ell}$ via

$c_{m, \ell}^{\dagger}=\sum_{a=0}^{m} A^{-1}(\ell)_{m, a} \tilde{c}_{a, \ell}^{*}, \quad c_{m, \ell}=\sum_{a=m}^{n-1} \tilde{c}_{a, \ell} A(\ell)_{a, m}$.

The (real, lower-triangular) matrix $A(\ell)$ is the only geometry-dependent aspect of this formalism. It is given in Ref. [40] for the disk and cylinder geometries.

The considerable advantage of the second-quantized formalism [33], especially for multiple LLs, lies in the fact that it gives us control over an algebra of " $\mathrm{ZM}$ generators" we arguably do not have in first quantization. It is also much more conducive to recursive schemes in particle number, which we will now heavily pursue. To this end, we introduce the following operators, which we will think of as ZM generators in a sense to be made precise:

$\hat{p}_{k}^{a, b}=\sum_{\ell} \tilde{c}_{a, \ell+k}^{*} \tilde{c}_{b, \ell}(a \geq b-k$ for disk geometry $)$,

and which is a generalization of the operator $\mathcal{O}_{d}$ introduced in Ref. [33] for multiple LLs.

The operators in Eq. (4) generate an algebra (via taking sums and/or products) that we denote by $\mathcal{Z}$. The significance of this algebra is manifold [31]. It allows for a definition of $\mathrm{CF}$ states recursive in particle number, quite distinct from the recently fashionable matrix-product presentation of $\mathrm{FQH}$ states [35,36], but it is, in essence a generalization of Read's expression of the Laughlin state through a nonlocal order parameter [53,54]. Indeed, the algebra allows for a microscopic definition [31] of a complete set of order parameters for CF states. In the present context, it will turn out that the algebra $\mathcal{Z}$ generates all possible ZMs when acting on the incompressible ground state. In that sense they are related to the first-quantized formalism discussed by Stone [55] for the Laughlin state, possible there because $\sum_{a=0}^{n-1} \hat{p}_{k}^{a, a}$ [which, for $n=1 \mathrm{LL}$, is really all Eq. (4) boils down to] has a simple first-quantized interpretation: It multiplies many-body wave functions with power-sum symmetric polynomials $p_{z}=\sum z_{i}^{k}[33,54]$. For multiple LLs, however, we need the full set $\hat{p}_{k}^{a, b}$, which does not have a straightforward first-quantized interpretation $[30,31]$.

Consider now Eq. (1). To resolve the "edge ambiguity" mentioned above, we define the Slater determinant by successively filling the state $\mu_{a, \ell}$ with lowest available $\ell+a$ that has lowest not-yet-occupied $a$. We seek to 
establish a parent Hamiltonian such that Eq. (1), which we now also suitably write $\left|\Psi_{n, \mathrm{p}, N}\right\rangle$, is a $\mathrm{ZM}$ of this Hamiltonian. Since general ZMs will describe edge excitations and, deeper in the bulk, quasihole excitations [56], one has the intuition [53] that $\tilde{c}_{a, \ell}\left|\Psi_{n, \mathrm{p}, N}\right\rangle$, is also a ZM of the Hamiltonian, namely, one describing a cluster of quasiholes of total charge 1 inserted into $\left|\Psi_{n, \mathrm{p}, N}\right\rangle$. Anticipating that this is so, then, with the properties of the $\hat{p}_{k}^{a, b}$ as advertised, we must be able to interpret this as a ZM generated by some combination of $\hat{p}_{k}^{a, b}$ on top of the reference state $\left|\Psi_{n, \mathrm{p}, N-1}\right\rangle$, or

$$
\tilde{c}_{a, \ell}\left|\Psi_{n, \mathrm{p}, N}\right\rangle=\hat{Z}_{n, \mathrm{p}, N, a, \ell}\left|\Psi_{n, \mathrm{p}, N-1}\right\rangle,
$$

where $\hat{Z}_{n, \mathrm{p}, N, a, \ell}$ is a suitable element of the algebra $\mathcal{Z}$. Indeed, the relation between $\hat{Z}_{n, \mathrm{p}, N, a, \ell}$ and the generators (4) was made explicit in Ref. [31], but will not be needed in the following.

Parent Hamiltonian for composite fermions.-We are now ready to present the following Hamiltonian,

$$
\begin{aligned}
H_{n, p} & =\sum_{J, r, a, b} E_{a, b, J}^{r} T_{a, b, J}^{r^{\dagger}} T_{a, b, J}^{r}, \\
T_{a, b, J}^{r} & =\sum_{x} x^{r} \tilde{c}_{a, J+x} \tilde{c}_{b, J-x},
\end{aligned}
$$

where $J$ runs over half-integer values with $J \geq-n$, $0 \leq r<2 p, 0 \leq a \leq b<n$. The $T_{a, b, J}^{r \dagger} T_{a, b, J}^{r}$ [57] are suitable generalizations of pseudopotentials, whose relation to Haldane pseudopotentials for $n=1$ was discussed in Ref. [33]. The $E_{a, b, J}^{r}$ are positive constants and may be used to enforce desirable spatial symmetries. We show in Ref. [40] that positive $E_{a, b, J}^{r}$ can always be chosen so as to render the resulting Hamiltonian local. The $T_{a, b, J}^{r}$ may also be replaced with new linearly independent combinations without affecting the ZM space. It is worth noting that the absence of a kinetic energy splitting between the first $n$ LLs is a feature that is realized in certain stackings of multilayer graphene [58-60].

For fermions, $T_{a, b, J}^{r}$ vanishes for even $r$ and $a=b$, giving $\mathrm{p} n^{2}$ different pseudopotentials at each pair-angular momentum $2 J$. Assuming disk geometry, we use the convention $\tilde{c}_{a, \ell} \equiv 0$ for $a+\ell<0$. A key observation is that the operators $T_{a, b, J}^{r}$ and $\hat{p}_{k}^{a, b}$ satisfy the following commutation relation:

$$
\begin{aligned}
{\left[T_{a, b, J}^{r}, \hat{p}_{k}^{a^{\prime}, b^{\prime}}\right]=} & \sum_{\tilde{r}=0}^{r}\left(\begin{array}{l}
r \\
\tilde{r}
\end{array}\right)\left(\frac{k}{2}\right)^{r-\tilde{r}} \\
& \times\left[(-1)^{r-\tilde{r}} T_{a, b^{\prime}, J-k / 2}^{\tilde{r}} \delta_{b, a^{\prime}}+T_{b^{\prime}, b, J-k / 2}^{\tilde{r}} \delta_{a, a^{\prime}}\right] .
\end{aligned}
$$

This justifies the notion that the $\hat{p}_{k}^{a, b}$ are $\mathrm{ZM}$ generators: The condition for $|\psi\rangle$ to be a ZM of the positive semidefinite Hamiltonian (6) reads $T_{a, b, J}^{r}|\psi\rangle=0$ for all $r, J, a$, $b$. The commutator (8) thus clearly vanishes within the ZM subspace. Therefore, any $\hat{p}_{k}^{a, b}$ acting on $|\psi\rangle$ immediately generates another $\mathrm{ZM}$, with angular momentum increased by $k$. In the following, we first wish to (i) establish that the Jain state $\left|\Psi_{n, \mathrm{p}, N}\right\rangle$ is a ZM of Eq. (6) and (ii) find all ZMs of Eq. (6).

We achieve these goals via a radical departure from established paradigms, i.e., not paying attention whatsoever to analytic clustering properties. We will do so by utilizing the properties of the second-quantized operator algebras given above and in the following. For part (i), we give a simple proof by induction in $N$ which extends that of Ref. [61]. We give the induction step first, assuming that $\left|\Psi_{n, \mathrm{p}, N-1}\right\rangle$ is known to be a ZM. One easily verifies that $T_{a, b, J}^{r}=\frac{1}{2} \sum_{\tilde{a}, \ell}\left[T_{a, b, J}^{r}, \tilde{c}_{\tilde{a}, \ell}^{*}\right] \tilde{c}_{\tilde{a}, \ell}$. We apply this to $\left|\Psi_{n, \mathrm{p}, N}\right\rangle$. Using Eq. (5) together with the fact that $\hat{Z}_{n, \mathrm{p}, N, a, \ell}$ is a ZM generator, i.e., $T_{a, b, J}^{r}$ annihilates Eq. (5), and that $\sum_{a, \ell} \tilde{c}_{a, \ell}^{*} \tilde{c}_{a, \ell}$ gives the total particle number $N$, yields $T_{a, b, J}^{r}\left|\Psi_{n, \mathrm{p}, N}\right\rangle=(N / 2) T_{a, b, J}^{r}\left|\Psi_{n, \mathrm{p}, N}\right\rangle$, or $T_{a, b, J}^{r}\left|\Psi_{n, \mathrm{p}, N}\right\rangle=0$ for $N>2$. So far, the only special property of the $T^{r}$ operators $(0 \leq r<2 \mathrm{p})$ that we have used is that $\hat{Z}_{n, \mathrm{p}, N, a, \ell}$ is a $\mathrm{ZM}$ generator as defined above. All that is left to do is to establish an induction beginning for $N=2$. Indeed, the $N=2$ state in the class of states $\left|\Psi_{n, \mathrm{p}, N}\right\rangle$ has the wave function $\left(z_{1}-z_{2}\right)^{2 \mathrm{p}}\left(\bar{z}_{1}-\bar{z}_{2}\right)$, or, in second quantization,

$$
\left|\Psi_{n, \mathrm{p}, 2}\right\rangle=\sum_{j}(-1)^{j}\left(\begin{array}{c}
2 \mathrm{p} \\
j
\end{array}\right) \tilde{c}_{1, j-1}^{*} \tilde{c}_{0,2 \mathrm{p}-j}^{*}|0\rangle .
$$

This state has angular momentum $2 \mathcal{J}=2 p-1$, and the only $T^{r}$ operators that could possibly not annihilate it are of the form $T_{0,1, \mathcal{J}}^{r}$. Acting with these operators produces

$$
\sum_{j}\left(j-\mathrm{p}-\frac{1}{2}\right)^{r}(-1)^{j}\left(\begin{array}{c}
2 \mathrm{p} \\
j
\end{array}\right)|0\rangle=0,
$$

for $r<2 \mathrm{p}$, since indeed [62] $\sum_{j=0}^{2 \mathrm{p}}(-1)^{j}\left(\begin{array}{c}2 \mathrm{p} \\ j\end{array}\right)(x-j)^{2 \mathrm{p}}=$ $(2 p)$ ! independent of $x$, such that taking $x$ derivatives implies Eq. (10).

Entangled Pauli principle (EPP).-_Having now established that the Jain state $\left|\Psi_{n, \mathrm{p}, N}\right\rangle$ is a ground state of the Hamiltonian $H_{n, \mathrm{p}}$, Eq. (6), we seek to understand the full ZM space of these Hamiltonians. This will, in particular, establish the densest $\mathrm{ZM}(\mathrm{s})$ of this Hamiltonian, whose existence is generally taken as the hallmark of incompressibility. The key to obtaining such results for Hamiltonians of the form (6) lies in the fact that there is a now wellestablished $[21,22,33,61]$ general method to derive necessary conditions, in the form of "EPPs" [22], on the "root 
states" for ZMs of such Hamiltonians. These root states encode the DNA of the incompressible fluids. Using these techniques we now establish that a complete set of ZMs for $H_{n, \mathrm{p}}$ is of the form (1), with the IQH state $\Phi_{n}$ replaced by $\mathcal{S}_{n}$, a generic Slater determinant with definite occupancies in $n$ Landau-/ $\Lambda$ Ls. That indeed such states are ZMs follows easily from the fact that the $\hat{p}_{k}^{a, b}$ are $\mathrm{ZM}$ generators, together with the convenient property that they commute [31] with the Laughlin-Jastrow flux-attachment operator. Acting on Eq. (1), the $\hat{p}_{k}^{a, b}$ may thus be thought of as acting directly on the IQH factor $\Phi_{n}$, thus, on $\Lambda \mathrm{L}$ degrees of freedom. It is easy to see that any $\mathcal{S}_{n}$ can be generated out of $\Phi_{n}$ by acting with appropriate products of $\hat{p}_{k}^{a, b}$ 's.

Consider now the expansion of any ZM $|\psi\rangle$ into $\Lambda \mathrm{L}$ Slater determinants:

$$
|\psi\rangle=\sum C_{\left(a_{1}, \mathfrak{r}_{1}\right), \ldots,\left(a_{N}, \mathfrak{r}_{N}\right)} \tilde{d}_{a_{1}, \mathfrak{r}_{1}}^{*} \cdots \tilde{d}_{a_{N}, \mathfrak{r}_{N}}^{*}|0\rangle,
$$

where we introduce $\tilde{d}_{a, \mathfrak{r}}=\tilde{c}_{a, \mathfrak{r}-a}, \tilde{d}_{a, \mathfrak{r}}^{*}=\tilde{c}_{a, \mathfrak{r}-a}^{*}$, with labels that refer to a "pseudo-guiding-center" $\mathfrak{R}=\sum_{a, \mathfrak{r}} \mathfrak{r} \tilde{d}_{a, \mathfrak{r}}^{*} \tilde{d}_{a, \mathfrak{r}}$ [63]. This renders $\mathfrak{r}$ to be non-negative, just as $a$. We define terms in the expansion (11) as "nonexpandable" [33] if the action with every possible "expansion" operator of the form $\tilde{d}_{a_{1}^{\prime}, \mathfrak{r}_{1}-x}^{*} \tilde{d}_{a_{2}^{\prime}, \mathfrak{r}_{2}+x}^{*} \tilde{d}_{a_{1}, \mathfrak{r}_{1}} \tilde{d}_{a_{2}, \mathfrak{r}_{2}}, \mathfrak{r}_{1} \leq \mathfrak{r}_{2}, x>0$ leads to a term with zero coefficient. The root state of $|\psi\rangle,|\psi\rangle_{\text {root }}$, is now defined as that part of the expansion (11) consisting only of nonexpandable terms. $|\psi\rangle_{\text {root }}$ so defined is necessarily nonvanishing due to the finite dimensionality of the subspace of given $\mathfrak{R}[22,64]$. As shown in Ref. [40], $|\psi\rangle_{\text {root }}$ is subject to the following EPP. (i) The $\mathfrak{r}$ values of any two occupied single-particle states differ at least by $2 p$. (ii) If they differ precisely by $2 p$, the root-level coefficients have the following antisymmetry property in $\Lambda \mathrm{L}$ indices:

$$
C_{\ldots\left(a_{i}, \mathbf{r}_{i}\right),\left(a_{i+1}, \mathbf{r}_{i}+2 \mathrm{p}\right) \ldots}=-C_{\ldots\left(a_{i+1}, \mathbf{r}_{i}\right),\left(a_{i}, \mathbf{r}_{i}+2 \mathrm{p}\right) \ldots} .
$$

As in many known examples, the EPP immediately reveals the densest possible filling factor at which ZMs of the model (6) may exist. To this end, it is useful to translate the EPP into a language of $\mathrm{SU}(n)$ spins, where each spin carries the fundamental representation. We may think of the $\Lambda \mathrm{L}$ index of a particle as an $\mathrm{SU}(n)$ index, and of its $\mathfrak{r}$ index as the position in a one-dimensional lattice. Then, permissible root states must be (linear combinations of) product states associated with certain clusters, each cluster containing up to $n$ particles. Within each cluster, particles are $2 p$ sites apart, and the "spin" wave function of each cluster is totally antisymmetric. This renders the largest possible cluster an "SU $(n)$ singlet" of $n$ spins [Fig. 1(a)], and clusters must be separated by at least $2 p+1$ sites. It is easy to see that the densest possible root state is just a product of such clusters at a filling factor of $n /(2 n p+1)$. There are thus no ZMs whose filling factor can exceed this value in the thermodynamic limit, and the corresponding Jain state

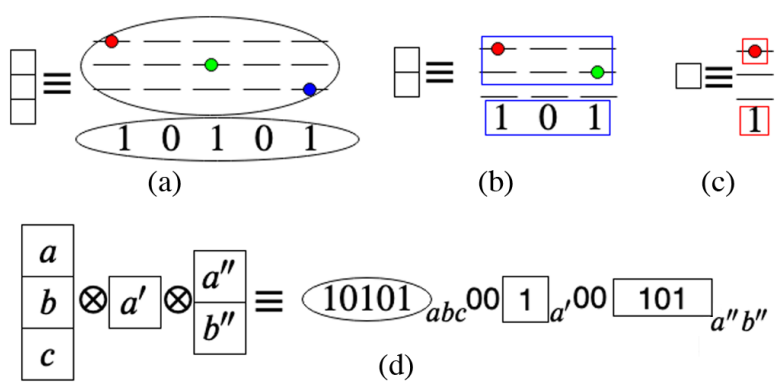

FIG. 1. Graphic representation of the clusters emerging at root level $|\Psi\rangle_{\text {root }}$. Panels (a)-(c) show the individual building blocks for root states assuming $n=3, \mathrm{p}=1$, such that the underlying group structure is SU(3) (see text). The oval (a) denotes a singlet. Panel (d) represents a sample root structure. Clusters generally consist of up to $n$ particles at distance $2 p$, totally antisymmetric in $\Lambda \mathrm{L}$ indices. Clusters are further characterized by the $\Lambda \mathrm{L}$ indices that are occupied, and must be mutually separated by at least $2 p+1$ orbitals.

just satisfies this bound. One can, more generally, show [40] that the number of possible root states sets an upper bound for the number of ZMs present in each angularmomentum- $/ \mathfrak{R}$ sector. A state counting argument shows, in turn, that the number of $\mathrm{CF}$ states of the form (Jastrow factor) $\times \mathcal{S}_{n}$ precisely saturates this bound [40]. Therefore, such CF states form a complete set of ZMs of Eq. (6). It is further easy to see that the counting of such CF states in a given angular momentum sector (relative to a minimum angular momentum CF state) coincides with the number of modes in the expected edge theory of $n$ branches of chiral fermions or bosons. This is pleasingly consistent with the fact that these ZMs are all generated by the application of the bosonic "density modes" (4) on the reference state (1), and that these modes have the simple action on the Slater parts of CF states stated above. The Hamiltonians constructed here are thus true representatives of the ZM paradigm discussed initially. Detailed numerical verification of this result is reported in Ref. [40].

Emergent $\mathrm{SU}(n)$ symmetry.-In essence, the above establishes that root states $|\Psi\rangle_{\text {root }}$ come as products of representations of $\mathrm{SU}(n)$. Indeed, an underlying $\mathrm{SU}(n)$ symmetry is present not only at root level, but is an emergent property of the full ZM space. To make this symmetry readily visible, we write the commutation relations of the zero-mode generators [31]:

$$
\left[\hat{p}_{k}^{a, b}, \hat{p}_{k^{\prime}}^{b^{\prime}, a^{\prime}}\right]=\delta_{b, b^{\prime}} \hat{p}_{k+k^{\prime}}^{a, a^{\prime}}-\delta_{a, a^{\prime}} \hat{p}_{k+k^{\prime}}^{b^{\prime}, b}
$$

In a cylindrical geometry, where there is no constraint on the subscript $k$, the above commutator is just the loop algebra of $\mathrm{SU}(n)$. In particular, for $k=k^{\prime}=0$, we recover the algebra of $\mathrm{SU}(n)$ itself [65]. For the disk, we have the constraint $a \geq b-k$, and the operators $\hat{p}_{b-a}^{a, b}$ still realize an $\mathrm{SU}(n)$ subalgebra. Therefore, the invariance of the ZM space under the infinite-dimensional algebra of zero-mode 
generators implies, in particular, its invariance under an $\mathrm{SU}(n)$ subalgebra. In view of the intimate connection between the ZM generators $\hat{p}_{k}^{a, b}$ and the edge effective theory, it is not surprising that this $\mathrm{SU}(n)$ structure has long been associated to Jain CF states based on field theoretic grounds and/or variational constructions [66]. Through the present work, this structure becomes an exact feature of a solvable microscopic model for the Jain CF phases (though, it will, of course, not remain exact under generic perturbations). For the special case $n=2$, the similarity with the findings of Ref. [22] strongly suggests that much of the formalism presented here can be carried over to a rich class of "partonlike" states [3,7,29], which offer a large playground for the exploration of non-Abelian topological phases [30]. We leave this as an interesting challenge for future work.

Conclusions. - The theory of the FQH effect traditionally rests on two pillars: (i) quantum-many-body wave functions and (ii) effective field theories. Hamiltonians that are exactly solvable and fall into the ZM paradigm provide a transparent connection between these pillars. The incredibly detailed link between the microscopics and effective field theory provided by edge mode counting has no counterpart in any other area of strongly correlated physics in more than one dimension. Even among the myriad phases of the $\mathrm{FQH}$ regime, the definitive parent Hamiltonians satisfying this paradigm cannot always be given. This used to be the situation for the most important class of phases in this regime, those described by Jain CF states. The present work exposes the underlying reasons for this and solves this problem by departing considerably from traditional Hamiltonian construction principles. The latter seek to describe a suitable few-body density matrix via analytic clustering principles. This cannot be done adequately in the case at hand. Instead, we circumvent this problem by an algebraic characterization of few-body correlations in a suitable operator framework. Apart from giving a satisfying solution to the lack of parent Hamiltonians for Jain states [38], we expect the formalism presented here to be of profound value in the exploration of vast classes of more complicated mixed-LL wave functions realizing rich non-Abelian physics, as well as to complement traditional lowest-LL methods. We are hopeful that this angle will inspire exciting future developments.

The work of S.B. was supported by the VILLUM FONDEN via the Centre of Excellence for Dirac Materials (Grant No. 11744) and the European Research Council under the European Union's Seventh Framework ERS-2018-SYG 810451 HERO.G. O. would like to acknowledge partial support by the DOE Grant No. DESC0020343 and the Clark Way Harrison Professorship at Washington University. We would like to thank J. K. Jain for insightful discussions.
[1] D. C. Tsui, H. L. Stormer, and A. C. Gossard, Phys. Rev. Lett. 48, 1559 (1982).

[2] R. B. Laughlin, Phys. Rev. Lett. 50, 1395 (1983).

[3] J. K. Jain, Phys. Rev. B 40, 8079 (1989).

[4] B. I. Halperin, Helv. Phys. Acta 56, 75 (1983).

[5] G. W. Moore and N. Read, Nucl. Phys. B360, 362 (1991).

[6] M. Milovanovic and N. Read, Phys. Rev. B 53, 13559 (1996).

[7] X. G. Wen, Int. J. Mod. Phys. B 04, 239 (1990).

[8] A. Lopez and E. Fradkin, Phys. Rev. B 44, 5246 (1991).

[9] G. Murthy and R. Shankar, Field theory of the fractional quantum hall effect, in Composite Fermions, edited by $\mathrm{O}$. Heinonen (World Scientific, Singapore, 1998), pp. 254-306.

[10] F. D. M. Haldane, Phys. Rev. Lett. 51, 605 (1983).

[11] S. A. Trugman and S. Kivelson, Phys. Rev. B 31, 5280 (1985).

[12] N. Read, Phys. Rev. B 79, 245304 (2009).

[13] N. Read, Phys. Rev. B 79, 045308 (2009).

[14] J. K. Jain, Phys. Rev. Lett. 63, 199 (1989).

[15] Y. Hu and J. K. Jain, Phys. Rev. Lett. 123, 176802 (2019).

[16] W. N. Faugno, A. C. Balram, M. Barkeshli, and J. K. Jain, Phys. Rev. Lett. 123, 016802 (2019).

[17] G. A. Csáthy and J. K. Jain, Nat. Phys. 15, 883 (2019).

[18] E. H. Rezayi and A. H. MacDonald, Phys. Rev. B 44, 8395 (1991).

[19] J. K. Jain, S. A. Kivelson, and N. Trivedi, Phys. Rev. Lett. 64, 1297 (1990).

[20] G. J. Sreejith, M. Fremling, G. S. Jeon, and J. K. Jain, Phys. Rev. B 98, 235139 (2018).

[21] L. Chen, S. Bandyopadhyay, and A. Seidel, Phys. Rev. B 95, 195169 (2017).

[22] S. Bandyopadhyay, L. Chen, M. T. Ahari, G. Ortiz, Z. Nussinov, and A. Seidel, Phys. Rev. B 98, 161118(R) (2018).

[23] J. K. Jain, Adv. Phys. 41, 105 (1992).

[24] B. A. Bernevig and F. D. M. Haldane, Phys. Rev. Lett. 100, 246802 (2008).

[25] S. H. Simon, E. H. Rezayi, N. R. Cooper, and I. Berdnikov, Phys. Rev. B 75, 075317 (2007).

[26] B. A. Bernevig and F. D. M. Haldane, Phys. Rev. B 77, 184502 (2008)

[27] X.-G. Wen and Z. Wang, Phys. Rev. B 77, 235108 (2008).

[28] X.-G. Wen and Z. Wang, Phys. Rev. B 77, 235108 (2008).

[29] X. G. Wen and Q. Niu, Phys. Rev. B 41, 9377 (1990).

[30] M. T. Ahari et al. (to be published).

[31] L. Chen, S. Bandyopadhyay, K. Yang, and A. Seidel, Phys. Rev. B 100, 045136 (2019).

[32] F. D. M. Haldane, Phys. Rev. Lett. 107, 116801 (2011).

[33] G. Ortiz, Z. Nussinov, J. Dukelsky, and A. Seidel, Phys. Rev. B 88, 165303 (2013).

[34] J. Dubail, N. Read, and E. H. Rezayi, Phys. Rev. B 86, 245310 (2012).

[35] M. P. Zaletel and R. S. K. Mong, Phys. Rev. B 86, 245305 (2012).

[36] B. Estienne, Z. Papić, N. Regnault, and B. A. Bernevig, Phys. Rev. B 87, 161112(R) (2013).

[37] M. Schossler et al. (to be published).

[38] While we focus on fermions here for brevity, generalization of our results to bosons, $p$ half-odd integer, is straightforward. 
[39] F. Bagarello, Theor. Math. Phys. 193, 1680 (2017).

[40] See Supplemental Material at http://link.aps.org/ supplemental/10.1103/PhysRevLett.124.196803 for for proofs of the locality of the Hamiltonian and of the completeness of the zero modes identified in the main text, as well as some numerical tests, which includes Refs. [10,33,41-52].

[41] E. J. Bergholtz and A. Karlhede, Phys. Rev. Lett. 94, 026802 (2005).

[42] E. J. Bergholtz and A. Karlhede, J. Stat. Mech. (2006) L04001.

[43] E. J. Bergholtz and A. Karlhede, Phys. Rev. B 77, 155308 (2008).

[44] E. J. Bergholtz, J. Kailasvuori, E. Wikberg, T. H. Hansson, and A. Karlhede, Phys. Rev. B 74, 081308(R) (2006).

[45] A. Seidel, H. Fu, D.-H. Lee, J. M. Leinaas, and J. Moore, Phys. Rev. Lett. 95, 266405 (2005).

[46] A. Seidel and D.-H. Lee, Phys. Rev. Lett. 97, 056804 (2006).

[47] A. Seidel and K. Yang, Phys. Rev. Lett. 101, 036804 (2008).

[48] A. Seidel, Phys. Rev. Lett. 105, 026802 (2010).

[49] A. Seidel and K. Yang, Phys. Rev. B 84, 085122 (2011).

[50] J. Flavin and A. Seidel, Phys. Rev. X 1, 021015 (2011).

[51] J. Flavin, R. Thomale, and A. Seidel, Phys. Rev. B 86, 125316 (2012).

[52] Z. Zhou, Z. Nussinov, and A. Seidel, Phys. Rev. B 87, 115103 (2013).

[53] N. Read, Phys. Rev. Lett. 62, 86 (1989).
[54] T. Mazaheri, G. Ortiz, Z. Nussinov, and A. Seidel, Phys. Rev. B 91, 085115 (2015).

[55] M. Stone, Phys. Rev. B 42, 8399 (1990).

[56] To justify the term "excitation," a confining potential that goes as some power of total angular momentum could be added.

[57] The Hermitian conjugates $\tilde{c}_{a, j}^{\dagger}$ appearing in this expression are related to the "pseudoconjugates" $\tilde{c}_{a, j}^{*}$ via $\tilde{c}_{a, j}^{\dagger}=$ $\left(\tilde{c}_{a, j}\right)^{\dagger}=A_{c a}^{-1} A_{c b}^{-1} \tilde{c}_{b, j}^{*}$.

[58] Y. Zhang, Z. Jiang, J. P. Small, M. S. Purewal, Y.-W. Tan, M. Fazlollahi, J. D. Chudow, J. A. Jaszczak, H. L. Stormer, and P. Kim, Phys. Rev. Lett. 96, 136806 (2006).

[59] Y.-H. Wu, T. Shi, and J. K. Jain, Nano Lett. 17, 4643 (2017).

[60] Y. Barlas, K. Yang, and A. H. MacDonald, Nanotechnology 23, 052001 (2012).

[61] L. Chen and A. Seidel, Phys. Rev. B 91, 085103 (2015).

[62] S. M. Ruiz, Math. Gaz. 80, 579 (1996).

[63] We specialize to the disk geometry here. For the cylinder, $\tilde{d}_{a, \mathfrak{r}}=\tilde{c}_{a, \mathfrak{r}}$ (see Ref. [40]). For the disk, the "ordinary" (Hermitian) guiding-center is $\sum_{a, \mathfrak{r}} \mathfrak{x} c_{a, \mathfrak{r}-a}^{\dagger} c_{a, \mathfrak{r}-a}$.

[64] We assume $|\psi\rangle$ to be an eigenstate of both angular momentum as well as $\mathfrak{R}$, as these operators commute with one another and with $H_{n, p}$.

[65] This is still true for the half-infinite cylinder, where the constraint is $k \geq 0$.

[66] N. Read, Phys. Rev. Lett. 65, 1502 (1990). 